\title{
Un cas de corrosion biologique en eau de bassin portuaire
}

\author{
A case study of a biological corrosion in ports basin
}

par M. El Haji, A. Abdelgader, C. Louis

Groupe de Recherche en Génie Civil, Université du Havre

\section{G. Breton}

Muséum d'Histoire Naturelle, Le Havre

The role of sulfate-reducing bacteria on the perforative corrosion of soft steel is now one of the principal areas of research undertaken in the field of microbiological corrosion. Frequently, the sulfate-reducing bacteria (SRB) are involved in the perforative corrosion within an anaerobic environment, although they might be present in an aerated environment within a biofilm as well. The influence of the SRB on the corrosion is not the only harmful effect. However, the growth of the bacteria can produce the compound $\mathrm{H}_{2} \mathrm{~S}$, which is highly toxic to most living beings. Since $\mathrm{H}_{2} \mathrm{~S}$ is one of the major products of the SRB's metabolism, certain industrial accidents can result from their presence. The aim of this work is to show, by counting of the SRB, the important contribution of these micro-organisms and others, such as iron bacteria and sulfo-bacteria, to the corrosion of soft steel, and therefore to the damage of fixed portuary installations.

\section{I INTRODUCTION}

Les micro-organismes jouent un rôle considérable dans la nature en intervenant dans de multiples cycles biologiques. De nombreuses espèces bactériennes jouent un rôle bénéfique et participent à la biodégradation des polluants ou à la synthèse de substances valorisables. D'autres au contraire ont une action néfaste et peuvent être considérées comme responsables de la dégradation de matériaux tels que les ouvrages portuaires [5], [6].

D'importantes dégradations ayant été observées au niveau d'ouvrages en acier, on étudie alors ce phénomène pour chercher les causes des altérations constatées. Pour ce faire on examine l'intervention des bactéries du cycle du soufre.

Au cours de la minéralisation de la biomasse morte, le soufre, comme les éléments essentiels (carbone, oxygène, azote), se trouve sous différentes formes, soit lié à différents composés organiques, soit libre, sous forme inorganique, et alors de l'état réduit à l'état oxydé. En conditions anaérobiès, le sulfure est la forme finale et énergétiquement stable du cycle du soufre.

\section{II — CYCLE BIOLOGIQUE DU SOUFRE}

Le soufre est un élément très répandu sous diverses formes solides, gazeuses ou dissoutes dans les eaux (soufre organique ou minéral à différents niveaux d'oxydation, acide sulfhydrique et sulfures, soufre, thiosulfates, sulfites, sulfates etc.). Il joue un rôle important dans les phénomènes biologiques. De nombreux micro-organismes interviennent dans le cycle du soufre et sont capables suivant les conditions du milieu de réaliser de multiples transformations moléculaires (oxydation ou réduction). Parmi ces micro-organismes, on distingue trois groupes qui sont respectivement capables :

— de minéraliser le soufre (exemple : Clostridium) ;

— d'oxyder le soufre (les sulfobactéries) ;

— de réduire le soufre (les sulfato-réducteurs).

\section{III — PRÉSENTATION DE LA ZONE D'ÉTUDE}

Le bassin et sas fluvial sont des bassins à flot anciens. Le niveau de l'eau est constant, hormis un marnage de 1 à $1,50 \mathrm{~m}$ entre les vives-eaux et les mortes-eaux.

Le bassin fluvial est un bassin de plan d'eau rectangulaire allongé Est-Ouest. De $550 \mathrm{~m}$ de long et d'environ $65 \mathrm{~m}$ de large, il est isolé des plans d'eau aval, à l'Ouest (sas fluvial) et amont, à l'Est (bassin Vétillart). Des eaux peuvent cependant percoler au travers des enrochements, mais semble-t-il, sans autoriser le passage de la macrofaune.

La nature du sédiment dans le bassin, étant très riche en matières organiques, elle est alors noire, putride à odeur nauséabonde.

La température de l'eau varie de $5^{\circ} \mathrm{C}$ en hiver à $18^{\circ} \mathrm{C}$, exceptionnellement $20^{\circ} \mathrm{C}$ à la belle saison. La salinité est de 
$27 \%$ dans le bassin fluvial ; les observations montrent de nombreuses combinaisons possibles de stratification d'eau de salinité et de température différentes (communication orale).

\section{IV — CONTEXTE THÉORIQUE}

La physiologie des BSR (bactéries sulfato-réductrices) éclaire leur distribution et leur rôle dans le milieu naturel. Leur croissance requiert la présence de sulfate dissous et de substrats organiques dans un environnement anaérobie ; de telles conditions sont fréquentes dans les habitats aquatiques profonds. L'accumulation de matière organique dans ces zones, où l'accès de l'oxygène est limité par la solubilité de ce gaz dans l'eau, entraîne une dégradation de la biomasse par les communautés bactériennes anaérobies dont les BSR font partie. Utilisant les produits de fermentation, elles réalisent l'étape terminale de la dégradation. En général c'est la présence ou l'absence de sulfate qui détermine la réaction favorisée.

\section{V — HYPOTHÈSES DE TRAVAIL}

\section{- 5.1 Corrosion par les sulfato-réducteurs}

Ce sont Von Wolgozen Kühr et Van Der Vlugt [15] qui les premiers identifièrent les BSR comme étant associées à des cas de corrosion d'acier dans les environnements anaérobies. Ils établirent alors la théorie désormais classique de la dépolarisation cathodique pour expliquer ce phénomène. Dans les conditions où les BSR sont susceptibles d'intervenir dans le processus de corrosion, c'est-à-dire à $\mathrm{pH}$ neutre en milieu anaérobie, la réaction cathodique produit un surpotentiel d'hydrogène qui polarise la surface et étouffe toute cellule électrolytique qui se serait formée. L'oxydation métabolique de I'hydrogène par les BSR (l'utilisation de l'hydrogène absorbé) provoquerait la dépolarisation cathodique, selon les réactions suivantes :

Réaction anodique $4 \mathrm{Fe} \rightarrow 4 \mathrm{Fe}^{2+}+8 \mathrm{e}$ -

Dissociation de l'eau $8 \mathrm{H}_{2} \mathrm{O} \rightarrow 8 \mathrm{OH}^{-}+8 \mathrm{H}^{+}$

Réaction cathodique $8 \mathrm{H}^{+}+8 \mathrm{e}-\rightarrow 4 \mathrm{H} 2$

Dépolarisation cathodique $\mathrm{SO}^{-}+8 \mathrm{H} \rightarrow 4 \mathrm{H}_{2} \mathrm{O}+\mathrm{S}^{2-}$

Produits de corrosion $\mathrm{H}_{2} \mathrm{~S}+\mathrm{Fe}^{2+} \rightarrow \mathrm{FeS}+2 \mathrm{H}^{+}$

$$
2 \mathrm{OH}^{+}+3 \mathrm{Fe}^{++} \rightarrow 3 \mathrm{Fe}(\mathrm{OH})_{2}
$$

Soit globalement: $\mathrm{SO}_{4}{ }^{2-}+4 \mathrm{Fe}+4 \mathrm{H}_{2} \mathrm{O} \rightarrow \mathrm{F}_{2} \mathrm{~S}+3 \mathrm{Fe}(\mathrm{OH})_{2}$ $+2 \mathrm{OH}$

Le schéma de la figure 1 met en évidence toutes les réactions dues aux ferrobactéries et aux BSR mais celles dues aux sulfobactéries n'interviennent pas directement dans le processus de la corrosion mais modifient le milieu environnant.

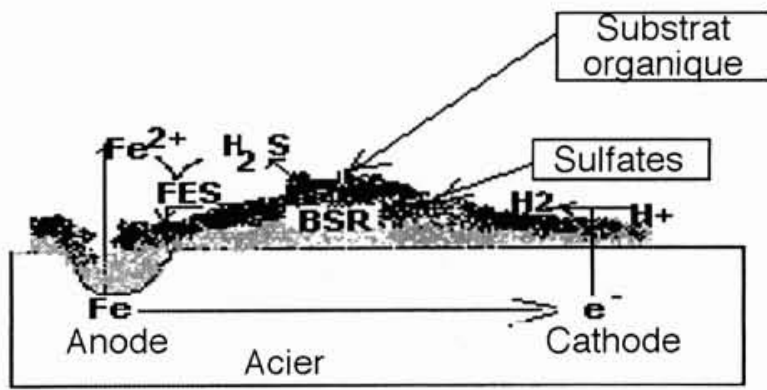

1. Mécanisme d'action des BSR dans la corrosion anaérobie selon la théorie de la dépolarisation cathodique, prenant en compte une réduction du sulfate avec des substrats organiques.

\subsection{BSR et corrosion dans l'environnement}

La nécessité presque absolue d'une colonisation directe du métal par les BSR a été reconnue dans de nombreux cas. Les travaux de Bryant et al. [4] avec des populations diversifiées naturelles de BSR par exemple, montraient que l'initiation de la corrosion coöncidait exactement avec la fixation d'un biofilm sur l'acier (fig.2).

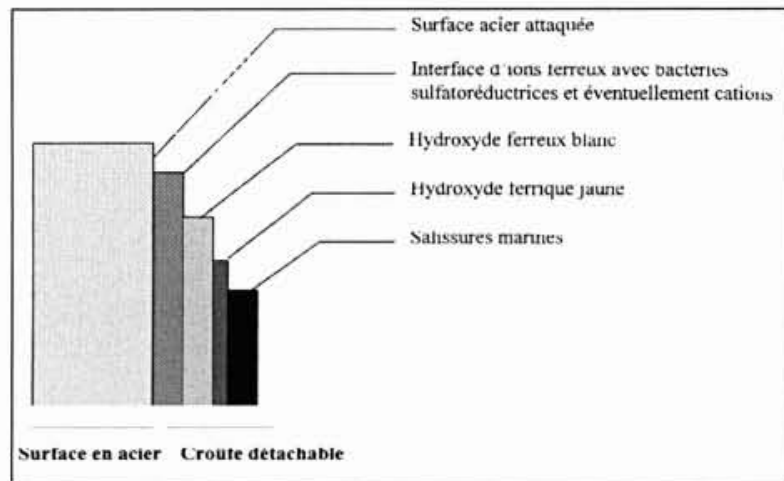

2. Formation du biofilm sur la surface d'acier

La présence de soufre élémentaire a été remarquée dans certains cas, à la périphérie des piqûres liées à l'action des BSR [9]. De fortes corrosions d'acier ont été observées en présence de soufre élémentaire, ce qui pourrait expliquer la corrosion rapide observée sur le terrain quand les conditions environnementales fluctuent de l'aérobiose à l'anaérobiose [16]. L'oxydation chimique ou bactérienne de sulfure pourrait alors conduire à la formation de soufre ou de polysulfures également corrosifs.
Tableau 1. Les différents sulfures de fer, d'après Smith et Miller (1975).

\begin{tabular}{|c|c|c|}
\hline Composé & Formule & $\begin{array}{c}\text { Variation } \\
\text { stoechiométrique }\end{array}$ \\
\hline Mackinawite & $\mathrm{Fe}(1+\mathrm{x}) \mathrm{S}$ & $\mathrm{x}=0,055$ à 0,064 \\
FeS cubique * & $\mathrm{FeS}$ & - \\
Pyrrhotite & $\mathrm{Fe}(1-\mathrm{x}) \mathrm{S}$ & $\mathrm{x}=0,14$ à 0,00 \\
Greigite & $\mathrm{Fe}_{3} \mathrm{~S}_{4}$ & - \\
Smythite & $\mathrm{Fe}(3+\mathrm{x}) \mathrm{S}_{4}$ & $\mathrm{x}=0$ à 0,25 \\
Marcasite & $\mathrm{FeS}_{2}$ & S-déficient \\
Pyrite & $\mathrm{FeS}_{2}$ & S-ou Fe-déficient \\
\hline
\end{tabular}

* le FeS cubique n'existe pas à l'état naturel 

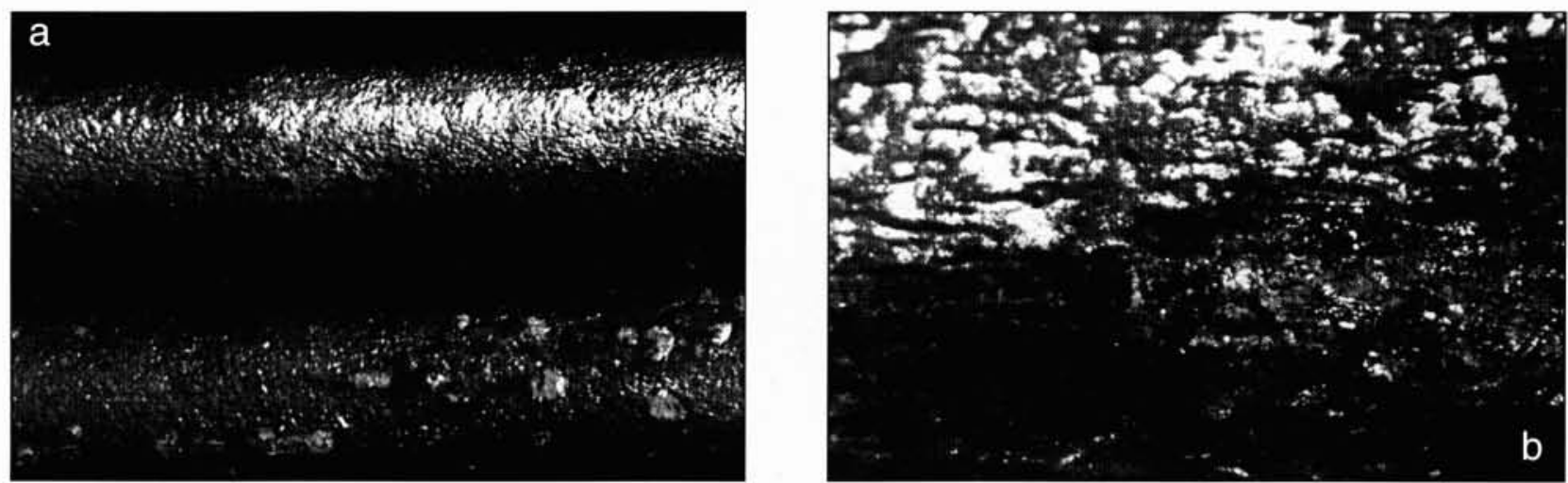

3. Développement de pyrite sur les barres d'acier doux

a) Comparaison avec une barre témoin après un mois d'implantation ; b) Granules de pyrite (source de sulfures de fer)

En conditions anaérobies, la solubilité du soufre élémentaire est influencée par la concentration en sulfure, le pH et la température, et seul le soufre dissous (présent sous l'une des différentes formes indiquées dans le tableau 1) a une action corrosive [13].

\section{VI $\square$ FACTEURS DE RISQUES DE CORROSION MICROBIENNE PAR LES BSR}

Les différents facteurs favorisant le développement des BSR sont regroupés dans le tableau 2.

\section{VII — DÉMARCHE EXPÉRIMENTALE}

Pour mettre en évidence les phénomènes de corrosion par les BSR, une dizaine de barres en acier doux ont été installées dans le bassin fluvial. Les premières observations au microscope optique, au bout du premier mois d'expérimentation, ont révélé la présence de pyrite (l'une des formes de sulfure de fer supposée utilisable par les BSR) sur la barre implantée jusqu'à l'interface eau-sédiment, comme le montrent les photographies de la figure 3 .

Au bout du deuxième mois, une recherche basée sur la méthode par ensemencement en milieux solides a été conçue directement après l'échantillonnage, ce qui permet le dénombrement des BSR.

\subsection{Méthode de dénombrement en milieux solides}

Dans un milieu synthétique adapté à l'action des bactéries sulfato-réductrices et contenant des sulfates, l'addition de fer, sous forme de sulfate ferreux, permet l'obtention de sulfure qui provoque un noircissement caractéristique du milieu [11].

Le milieu réactif est un milieu E de Postgate constitué de :

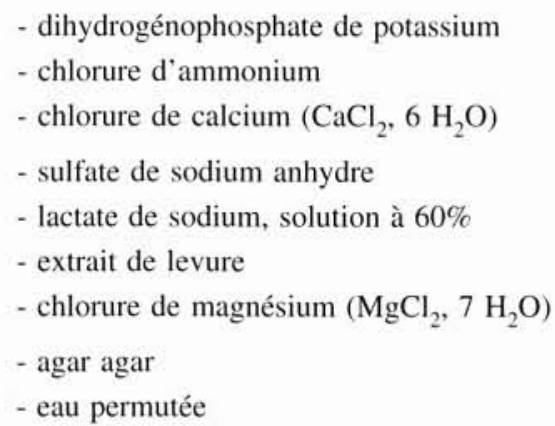

$0,5 \mathrm{~g}$

Les composants sont dissous dans l'eau permutée. On ajuste éventuellement le $\mathrm{pH}$ de façon à ce qu'après stérilisation, il soit de 7,6 . On répartit en tubes de $160 \times 16 \mathrm{~mm}$, à raison de $10 \mathrm{ml}$ par tube. Une stérilisation est réalisée en autoclave à $121^{\circ} \mathrm{C}$ pendant 15 minutes. Au moment de l'emploi, on régénère le milieu pendant 20 à 30 minutes au bain-marie bouillant. La solution est ensuite refroidie à 45 $60^{\circ} \mathrm{C}$ et on ajoute stérilement à chaque tube $0,1 \mathrm{ml}$ de chacune des trois solutions suivantes :

- solution de sulfate de fer à $5 \%$,

- solution d'acide ascorbique à $1 \%$,

- solution d'acide thioglycolique à $1 \%$.

\subsection{Mode opératoire}

Les deux opérations suivantes sont réalisées :

- Incorporation de $1 \mathrm{ml}$ de l'échantillon pur ou des différentes dilutions obtenues à partir de l'échantillon, dans le milieu liquifié, refroidi à $45^{\circ} \mathrm{C}$ et coulé en boîte de Pétri.

- Incubation à la température de $28-30^{\circ} \mathrm{C}$ pendant 15 jours.

A la suite de cela, on recherche et on dénombre à partir du troisième jour, l'apparition des colonies noires.

\begin{tabular}{|l|l|}
\hline $\begin{array}{l}\text { Facteurs favorables au } \\
\text { développement des BSR }\end{array}$ & \multicolumn{1}{c|}{ Désignation } \\
\hline - Anaérobiose & - milieu anoxique (absence totale de l'oxygène) \\
- Milieu réducteur & $-200 \mathrm{mV}$ à $300 \mathrm{mV}$ \\
- Présence de : & $-\mathrm{SO}_{4}^{2-}$ \\
& $-\mathrm{CO}_{3}^{2-}$ \\
& $-\mathrm{H}_{2}$ (hydrogène cathodique) \\
& - Matières organiques \\
& $-\mathrm{N}$ \\
- pH & - Proche de la neutralité \\
- Présence de $\mathrm{H}_{2} \mathrm{~S}$ & - Conditions très favorables à la corrosion du fer \\
\hline
\end{tabular}

Tableau 2. Facteurs favorisant l'attaque corrosive par les BSR. 

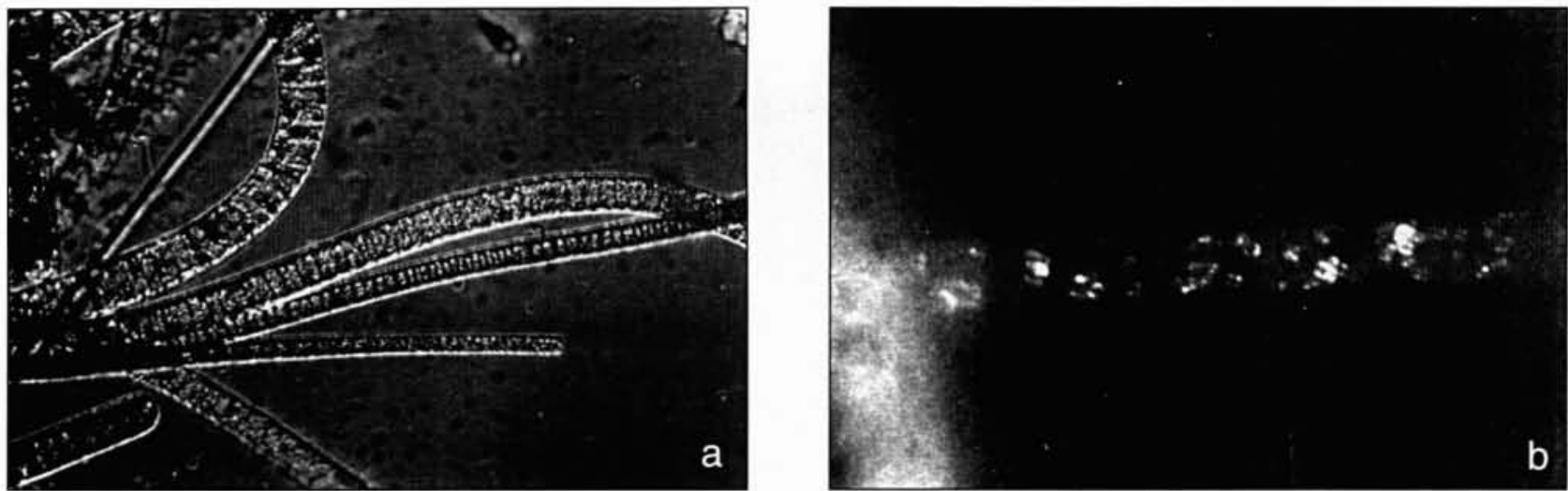

4. Bactéries filamenteuses à différents grossissements

a) Coupe optique des sulfo-oxydantes filamenteuses ; b) Beggiattoa accumulant des grains de soufre dans le cytoplasme.

\section{VIII @ MÉTABOLISMES BACTÉRIENS IMPLI- QUÉS DANS LA CORROSION}

\subsection{Cas des bactéries sulfo-oxydantes}

Les cas les plus spectaculaires de corrosion induite par les bactéries sulfo-oxydantes, essentiellement les thiobacilles, ont été observés sur des bétons ou des pierres [6], [7], [10], [12]. Toutefois dans certains environnements ce sont des métaux, ferreux ou non, qui subissent des conséquences de leur métabolisme. Ce métabolisme requiert une source de soufre ou de sulfure, qui peut être un dépôt atmosphérique, des pyrites ou de $\mathrm{l}^{\prime} \mathrm{H}_{2} \mathrm{~S}$ d'origine chimique ou biologique.

L'utilisation d'un composé soufré comme donneur d'électrons permet la croissance autotrophe des thiobacilles (en présence de $\mathrm{CO}_{2}$ comme source de carbone) avec la formation concomitante d'acide sulfurique. C'est cet acide qui est alors cause de la corrosion, éventuellement aggravée par les sels de fer produits si des pyrites servent de substrat. La détérioration de matériaux contenant du soufre natif peut être aussi causée par des thiobacilles qui l'utilisent alors directement [10].

Malgré leur préférence pour des conditions très différentes de celles favorisant la croissance des BSR, les bactéries sulfo-oxydantes coexistent souvent avec ces dernières, productrices de $\mathrm{l}^{\mathrm{H}} \mathrm{H}_{2} \mathrm{~S}$ indispensable à leur croissance.

Un autre type de sulfo-oxydant est représenté par les genres Beggiatoa et Thiotrix, bactéries filamenteuses accumulant le soufre dans leur cytoplasme sous forme de petits globules (comme le montre les photographies de la figure 4). On les trouve dans les habitats similaires à ceux des thioba- cilles, puisqu'elles ont besoin aussi de $\mathrm{H}_{2} \mathrm{~S}$ produit par les BSR. Bien qu'elles ne soient en général pas considérées comme directement responsables d'action sur la corrosion [14], elles peuvent former des amas visqueux, susceptibles d'abriter des BSR auxquelles elles sont liées pour des raisons métaboliques, comme les thiobacilles, et participer à la construction d'un biofilm plurispécifique.

\section{IX — INTERPRÉTATION DES RÉSULTATS}

Pour trois échantillons d'eau prélevés auprès du lieu d'implantation des barres en acier à l'interface eau-sédiment, des ensemencements en milieux solides, de $4 \mathrm{ml}$ et $0,4 \mathrm{ml}$ ont été effectués pour le dénombrement des bactéries sulfato-réductrices (tableau 3 ).

Ce dénombrement a permis de calculer le nombre de colonies de BSR par millilitre en utilisant l'équation suivante :

$$
\mathrm{Y}=\frac{\mathrm{X}}{\mathrm{V} \cdot \mathrm{d}}
$$

$\mathrm{Y}$ : nombre de colonies par $\mathrm{ml}$,

$\mathrm{X}$ : nombre de colonies trouvées dans la solution mère diluée à $10^{-1}$,

$\mathrm{V}$ : volume total utilisé pour l'isolement des colonies,

$\mathrm{d}$ : dilution choisie.

L'utilisation des résultats du tableau 3 a permis, selon l'équation précitée, d'obtenir 12,5 colonies par millilitre, ce qui permet d'avoir une idée assez claire sur l'abondance des BSR dans le site étudié.

Tableau 3. Dénombrements de colonies des BSR en milieu solide.

\begin{tabular}{|c|c|c|c|}
\hline Prise d'essai & $\begin{array}{c}\text { Nombre de colonies dans } \\
\text { l'échantillon } 1\end{array}$ & $\begin{array}{c}\text { Nombre de colonies dans } \\
\text { l'échantillon } 2\end{array}$ & $\begin{array}{c}\text { Nombre de colonies dans } \\
\text { l'échantillon } 3\end{array}$ \\
\hline $\begin{array}{l}\text { solution mère } \\
\qquad(4 \mathrm{ml})\end{array}$ & $\begin{array}{c}\text { milieu tout noir } \\
\text { (nappe de bactéries) }\end{array}$ & $\begin{array}{c}\text { milieu tout noir } \\
\text { (nappe de bactéries) }\end{array}$ & $\begin{array}{c}\text { milieu tout noir } \\
\text { (nappe de bactéries) }\end{array}$ \\
\hline $\begin{array}{l}\text { solution mère diluée à } 10^{-1} \\
(\mathbf{0 , 4} \mathbf{~ m l})\end{array}$ & 2 colonies & 0 colonie & I colonie \\
\hline
\end{tabular}




\section{$X$ — CONFIRMATION DE L'HYPOTHÈSE}

Dans le milieu naturel, les sulfures en milieu anoxique proviennent de la réduction des sulfates par les BSR. Les quantités de fer formées sont donc dépendantes de nombreux phénomènes, qui peuvent suivant les cas avoir une importance variable. Parmi ceux-ci citons la quantité et la qualité de la matière organique qui sédimente, la vitesse de dépôt, la quantité de sulfates disponibles ainsi que la température [2], [8]. [1]. La quantité de sulfure qui précipite peut être, quant à elle, fortement dépendante de la quantité de fer mobilisable.

La structure de la pyrite formée dépend en grande partie du degré de saturation de la solution dans laquelle elle précipite. Les vitesses de formation dépendent évidemment des réactifs initiaux. Cependant l'importance de la formation de ce produit constitue l'indice majeur confirmant la présence des BSR (de l'ordre de 12,5 colonies par millitres) dans le site étudié. Les BSR associées à l'action de plusieurs familles bactériennes, particulièrement les ferrobactéries et les sulfobactéries, génèrent des concrétions sous forme de tubercules très rapprochés provoquant des obstructions, quelquefois considérables et éventuellement des piqûres sousjacentes qui peuvent devenir perforantes.

\section{XI $\square$ CONCLUSION}

L'action des BSR, par attaque corrosive, est prépondérante et dominante dans le milieu étudié. Cependant, tous les micro-organismes du cycle du soufre sont très répandus mais leur activité dépend essentiellement de la nature du milieu, du $\mathrm{pH}$ et de la température. Cette étude montre donc qu'un enchaînement des mécanismes de corrosion suivants intervient :

- adsorption de nutriments par le biofilm, provoquant des différences d'aération et de concentrations chimiques, d'où la contribution des ferrobactéries dans la construction du biofilm (exemple: Gallionella et sphaerotillus),

- réduction de métabolites corrosifs par les BSR qui utilisent la pyrite comme source de sulfures de fer en produisant ainsi des $\mathrm{H}_{2} \mathrm{~S}$,

- oxydation des $\mathrm{H}_{2} \mathrm{~S}$ par les sulfobactéries (essentiellement les Thiobacillus et Beggiatoa). Les produits principaux de cette oxydation sont le soufre élémentaire et le sulfate.

\section{RÉFÉRENCES}

[I] Berner R.A. (1984).- Sedimentary pyrite formation : An update. Geochim. Cosmochim. Acta, 48, pp. 605-615.

[2] Berner R.A. and Westrich J.T. (1985).- Bioturbation and the early diagenesis of carbon and sulfur. Am . Journ. Sci., 285, pp. 193-206.

[3] Breton G., Girard A. and Lagardère J. P. (1995).- Espèces animales benthiques des bassins du port du Havre (Normandie, France) rares, peu connues ou nouvelles pour la région. Bull. Trim. Soc. Géol. Norm. et Amis du Muséum du Havre, t.82, fasc.3, pp. 1-28.

[4] Bryant R. D., Jansen W., Boivin J., Laishley E.J. and Costerton J.W (1991).- Affect of hydrogenas and mixed sulfate-reducing bacterial populations of the corrosion of steel. Appl. Environ. Microbiol. 57, pp. 2804 2809 .

[5] Divet L. (1996) - Activité sulfatique dans les bétons consécutive à l'oxydation des pyrites contenues dans les granulats. Synthèse bibliographique. Bulletin des Laboratoires des Ponts et Chaussées, 201, Réf. 3931. pp. 45-63.

[6] Divet L. (1996) - Etude des risques d'oxydation de la pyrite dans le milieu basique du béton. Bulletin des Laboratoires des Ponts et Chaussées, 204, Réf. 4019, pp. 97-107.

[7] Divet L. (1997) - Les réactions sulfatiques dans les bétons. Entretenir, réparer et renforcer les ouvrages en béton. Bulletin du Laboratoire Central des Ponts et Chaussées, 58, 24p.

[8] Goldhaber M.B and Kaplan I.R. (1980).- Mechanism of sulfure incorporation and isotope fractionation during early diagenesis in sediments of the Gulf of California. Rev. Mar. Chem., 3, pp. 83-104.

[9] Hamilton W.A. (1985).- Sulfate-reducing bacteria and anaerobic corrosion. Ann. Rev. Microbiol. 39, pp. 195-217.

[10] Postgate J.R. (1982).- Economic importance of sulphur bacteria. Phil. Trans. R. Soc. Lond. B 298, pp. 583-600.

[11] Rodier J. (1984),- L'analyse de l'eau, eaux naturelles, eaux résiduaires et eaux de mer : physico-chimie, bactériologie, biologie. Edition Dunod, Paris, $1383 \mathrm{p}$.

[12] Sand W. (1987).- Importance of hydrogen sulfide, thiosulfate and methylmercaptan for growth of thiobacilli during simulation of concrete corrosion. Rev. Appl. Environ. Microbiol., 53, pp. 1645-1648.

[13] Schalschl E. (1980).- Elemental sulfur as a corrodent in dearated, neutral aqueous solutions. Mat. Perf. 19, pp. 9-12.

[14] Tatnall R.E. (1981b). Fundamentals of bacteria induced corrosion. Mat. Perf. 20 (9), pp. 32-38.

[15] Von Wolgozen Kuihr C.A.H. and Van der Vlugt L.S. (1934).- The graphitization of cast iron as electrobiochemical process in anaerobic soils. Wat., Den Haag. 16, pp. 147-165

[16] Wang J. and Morse W. (1996) - Pyrite formation under conditions approximating those in anoxic sediments. Pathway and morphology. Rev. Mar. Chem., 52, pp. 99-121. 